\title{
Torsion modulus using the technique of mechanical spectroscopy in biomaterials ${ }^{\dagger}$

\author{
Carlos Alberto Fonzar Pintão*, Diego R. N. Correa and Carlos R. Grandini
} \\ School of Science, São Paulo State University (UNESP), 17033-360, Bauru, SP, Brazil
}

(Manuscript Received May 31, 2016; Revised November 25, 2016; Accepted December 30, 2016)

\begin{abstract}
This work presents a new alternative to studying and determining the shear or torsion modulus, $G$. For this purpose, a measuring system was constructed with a rotational motion sensor coupled to a torsion pendulum that allowed the determination of the angular position as a function of time. Through an equation derived from studies of mechanical spectroscopy and the theory of relaxation of materials, $G$ was calculated, and experiments were focused on validating it. The advantage of this technique, compared to other dynamical methods, is that it is not necessary to know the Poisson's ratio of the sample.
\end{abstract}

Keywords: Mechanical spectroscopy; Rotational inertia; Rotational motion sensor; Torsion modulus; Titanium

\section{Introduction}

The elastic modulus is a measure for the rigidity of a material and represents an important property in materials that could potentially be used as implants [1-5]. A significant difference in the elastic modulus of the implant compared to the bone can lead to bone loss and deployed material. Its value depends on the atomic bonds and the distances between the atoms in the crystalline lattice; therefore, this value is sensitive to the addition of elements, thermo-mechanical treatments and deformation processing. Traditionally, the elastic modulus is obtained by static (stress-strain curves) and dynamical (ultrasound or vibration) methods [6-8].

This paper establishes an alternative technique for determining the torsion modulus, $G$, for biomaterials. The main goal is to validate the equation for the calculation of $G$. This equation was derived from the study of mechanical spectroscopy [8] and the theory of relaxation of materials and depended on system rotational inertia, geometry parameters of the samples, frequency of oscillation and internal friction. The equation was developed to measure $G$. In this case, the analyzed samples show a constant circular cross-section, but the equation can be applied to other uniform cross-sections. These measurements are considered dynamic because, unlike conventional methods, they are not performed statically. In addition, since the value of $G$ modulus depends on the system oscillation frequency, this technique is of mechanical spectroscopy type and when applied to biomaterials is possible to identify

${ }^{*}$ Corresponding author. Tel.: +55 1431037654, Fax.: +55 1431036084

E-mail address: fonzar@fc.unesp.br

${ }^{\dagger}$ Recommended by Associate Editor Sang-Hee Yoon

(C) KSME \& Springer 2017 by comparative studies as the addition of a chemical element or thermal treatment modifies its rigidity $G$. The advantage of this technique when compared to other dynamical methods to obtain $E$ is that it is not necessary to know the Poisson's ratio of the sample. The elastic modulus, $E$ or $G$, depends on the atomic bonds in the crystalline lattice and is sensitive to the addition of other elements and thermo-mechanical treatments. Therefore, it is possible that Poisson's ratio be different if, instead of the present material, it was used, for example, Ti alloys with thermo-mechanical treatments. The latter fact may compromise the results of $E$ that depend on Poisson's ratio. As the technique presented here does not depend on Poisson's ratio, it cannot change the result of $G$. The cost of the measurement system is low and easy to use. It is not necessary to use a vacuum system and internal friction is small and it not influence the values of $G$ in a significant way.

Several techniques to determine the moment of inertia of a body can be found in the Refs. [9-14]. To determine the rotational inertia of the torsion pendulum, $I$, independent measurements were realized using a method developed in our research laboratory $[15,16]$.

The rotational inertia values were adjusted and modified for each measurement to obtain a different frequency response. A rotation sensor was used to study this movement in order to obtain a record of angular position as a function of time. The function of the "wave form" kind was adjusted to the experimental results, and then some parameters were determined that could provide information about the oscillation frequency, $f_{0}$, and the total internal friction of the system, $Q_{T}^{-1}=\delta / 2 \pi$. These parameters were used in the equation to calculate the $G$ modulus. Using an electromagnet, torsion was applied at one 

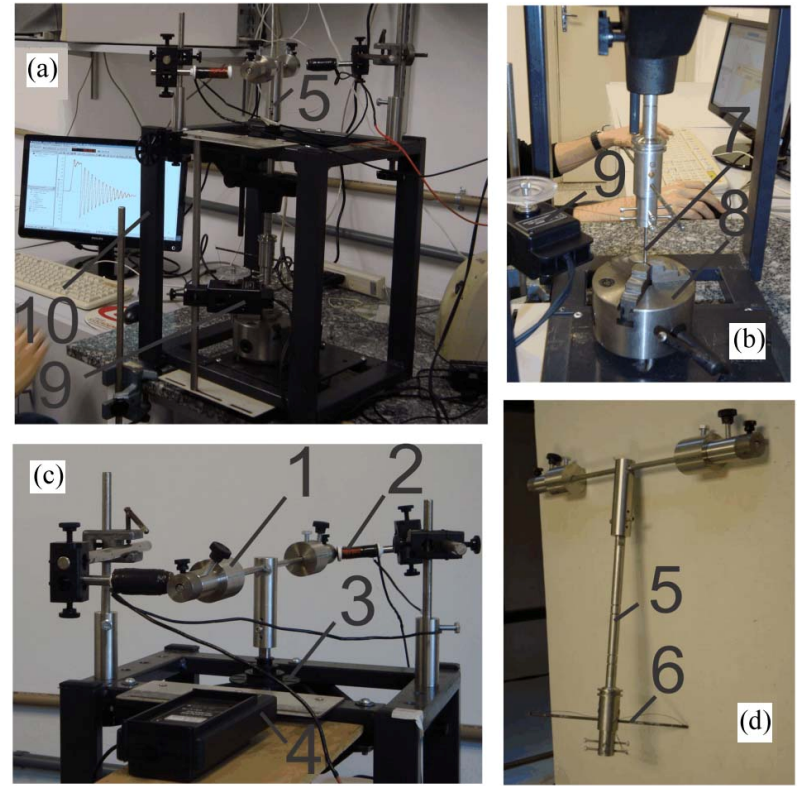

Fig. 1. Measurement system: (1) The masses of the torsion pendulum, (2) electromagnet, (3) three-step pulley in the position above the shaft of the Rotational system $(R S)$, (4) sensor of force $(F S)$ - PASCO: CI6537, (5) torsion pendulum, (6) $R S$ shaft rod, (7) sample, (8) sample fixing base, (9) Rotary motion sensor (RMS) - PASCO: CI-6538, (10) measurement system structure. (a) Complete view of the system; (b) detailed view of the system: $R M S$ and sample fixing base; (c) $F S$ and electromagnet; (d) pendulum.

end of a sample fixed to a pendulum where the other end was attached to the base of a static structure, which allowed the pendulum to oscillate freely until it stopped.

\section{Measurement of torsion modulus}

\subsection{Equation to determine the torsion modulus}

After applying a torque to the torsion pendulum and leaving it to oscillate freely, it stopped oscillating after a certain interval (see the system in Fig. 1). A rotational movement sensor was fixed to the base of the measuring system by attaching a wire to a pulley and connecting it to two rods, which formed part of the pendulum. This made it possible to register the pendulum's angular position $(\theta)$ as a function of time $(t)$. The generated curve in this record is the differential equation solution describing this movement, that is, $\theta=\theta(t)$. This equation, describing the oscillation [8] and based on Newton's second law and relaxation studies, is expressed by:

$$
I \ddot{\theta}+G_{0}(1+i \operatorname{tg} \varphi) \theta=0 .
$$

The symbols $I, G_{0}$ and $i$ represent the rotational inertia, the torsion constant of the wire or sample of the pendulum and the imaginary number $i$, respectively. In this case, by hypothesis, we discounted air resistance and the friction of the bearings on the rotational motion sensor and Rotational system $(R S)$ because they should not significantly influence the movement
[17]. The solution of the above equation is of the form, $\theta=\theta_{0} e^{i \omega^{*} t}$, where $\omega^{*}=\omega_{0}(1+i \delta / 2 \pi)$ and $\operatorname{tg} \varphi=\delta / \pi$.

Calculating the first and second derivative of $\theta=\theta_{0} e^{i \omega^{*} t}$ and substituting in Eq. (1), that is, $\dot{\theta}=\theta_{0} e^{i \omega^{*} t} i \omega$ and $\ddot{\theta}=\theta_{0} e^{i \omega^{*} t} i \omega^{*} i \omega^{*}=-\theta_{0} e^{i \omega^{*} t} \omega_{0}^{2}[1+i \delta / 2 \pi]^{2}$, then Eq. (1) is expressed as

$$
\begin{aligned}
& I\left[-\theta_{0} e^{i \omega^{*} t} \omega_{0}^{2}\left(1+i 2 \delta / 2 \pi-\delta^{2} / 4 \pi^{2}\right)\right]+ \\
& +G_{0}(1+i \operatorname{tg} \varphi) \theta_{0} e^{i \omega^{*} t}=0 .
\end{aligned}
$$

The real part of Eq. (2) is expressed as

$$
-I \omega_{0}^{2}+I \omega_{0}^{2} \delta^{2} / 4 \pi^{2}+G_{0}=0
$$

and the imaginary part by the equation is expressed as

$$
\left.-I \omega_{0}^{2} i \frac{\delta}{\pi}+G_{0} i \operatorname{tg} \varphi\right)=0
$$

From Eq. (3), it is written that

$$
G_{0}=I \omega_{0}^{2}\left(1-\frac{\delta^{2}}{4 \pi^{2}}\right)
$$

The latter equation suggests that $G_{0}$ is obtained by knowing the moment of inertia of the pendulum, $I$, the angular velocity, $\omega_{0}$, and the term $\delta / 2 \pi$. It is possible to alter the masses in the torsion rod of the pendulum, and it is possible to change $I$, which modifies the value $\omega_{0}$. This makes it possible to find $G_{0}$, depending on the frequency of the oscillation system. Therefore, we have a dynamic measure. On the other hand, if it is necessary to obtain $I$ using Eq. (5), a wire will be needed with a torsion value $G_{0}$, which can be experimentally determined [17]. To do this, simply use the force sensors and rotation and find the best fit of the force depending on the angular position. We know that for small angles of oscillation, in which the measuring system has a linear behavior, a straight line is the best fit to force versus angular position. The constant $G_{0}$ is determined by the slope of the force curve, $F$, as a function of the angular position, $\theta$, for the two quantities recorded with force sensors and rotation, respectively. With the value of the slope, $(\Delta F / \Delta \theta)$, multiplied by the radius of the pulley, $r_{P}$, you can determine $G_{0}$, which is

$$
G_{0}=\left(\frac{\Delta F}{\Delta \theta}\right) r_{p}=\frac{\text { applied torque }}{\theta},
$$

since the main objective is to determine the shear modulus, $G$, for a solid sample radius of a uniform circular section $R$ and length $L$. Therefore, when considering issues that take into account the resistance of the material [18-20], we can calculate the resistive torque to which the sample is submitted, that is, when it undergoes torsion by means of a pendulum or a torque is applied (see Figs. 1 and 2). This torque is calculated 


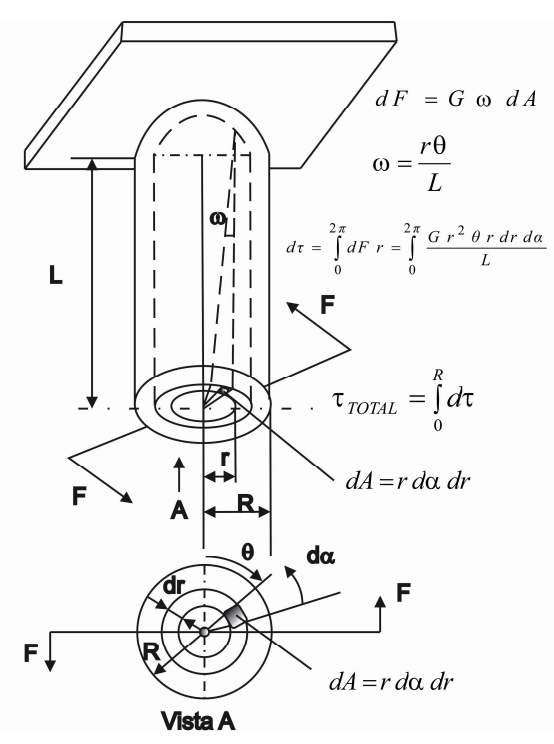

Fig. 2. Schematic drawing representing all quantities considered in order to obtain Eq. (7).

by the following equation:

$$
\text { resistive torque }=\int_{0}^{R} \int_{0}^{2 \pi} \frac{G r \theta r d r d \alpha}{L}=\frac{\pi}{2} G \theta \frac{R^{4}}{L}
$$

Fig. 2 shows a schematic drawing representing all the quantities considered in Eq. (7) that can facilitate the understanding of this result.

Comparing Eqs. (6) and (7), we arrive at

$$
G_{0}=\frac{\pi}{2} G \frac{R^{4}}{L}
$$

Thus, the magnitude of interest, $G$, is obtained by substituting Eq. (5) with Eq. (8). As a result,

$$
\begin{aligned}
& G=\frac{2}{\pi} \frac{L}{R^{4}} G_{0}=\frac{2}{\pi} \frac{L}{R^{4}} I \omega_{0}^{2}\left(1-\frac{\delta^{2}}{4 \pi^{2}}\right)= \\
& =\frac{32}{\pi} \frac{L}{d^{4}} I \omega_{0}^{2}\left(1-\frac{\delta^{2}}{4 \pi^{2}}\right) .
\end{aligned}
$$

If you replace $\omega^{*}=\omega_{0}(1+i \delta / 2 \pi)$ in the solution, $\theta=\theta_{0} e^{i \omega^{*} t}$, relative to the differential equation that describes the motion of the free pendulum [8], which we expressed, then

$$
\begin{aligned}
& \theta=\theta_{0} e^{i \omega_{0}\left(1+i \frac{\delta}{2 \pi}\right) t}=\theta_{0} e^{-\frac{\delta}{2 \pi} \omega_{0} t} e^{i \omega_{0} t}= \\
& =\theta_{0} e^{-\frac{\delta}{2 \pi} \omega_{0} t}\left(\cos \omega_{0} t+i \operatorname{sen} \omega_{0} t\right)
\end{aligned}
$$

In Eq. (10), a consideration of the real part arrives at the following:

$$
\theta=\theta_{0} e^{-\frac{\delta}{T} t} \cos \omega_{0} t
$$

The values of the $\delta$ and $\omega_{0}$ parameters were obtained from experimental results for the curve $\theta$ versus $t$, which was obtained using the rotational motion sensor software and the Pasco interface for a given value of $L, d$ and $I$. For this purpose, a fit function similar to Eq. (11) was devised with the Origin 7.0 software application by selecting a "wave form" function, such as:

$$
\phi=A \exp \left(-t / t_{0}\right) * \sin \left(\frac{\pi}{W} t-\frac{\pi t_{C}}{W}\right) .
$$

By comparing this with Eq. (11), we established a comparison that takes into account the parameters $t_{0}$ and $W$, obtained by fit of Eq. (12) to the experimental values. We were also able to obtain the $\delta$ and $\omega_{0}$ parameters:

$$
T=\frac{2 \pi}{\omega_{0}}=2 W \text { and } \delta=\frac{2 W}{t_{0}} \text {. }
$$

Finally, with the $W$ and $t_{0}$ parameters and Eq. (13), we calculated $(\delta / 2 \pi)^{2}$ and $\omega_{0}=2 \pi / T$, and using Eq. (9), we determined $G$.

\subsection{Method to determine rotational inertia}

We need the inertia moment $(M I)$ of the torsion pendulum $I$ because the objective of this work is to determine the shear modulus of materials using mechanical spectroscopy and to verify the validity of Eq. (9). We determined this by removing the sample and the electromagnetic system. In this situation, the same procedure was adopted as in Refs. $[15,16]$, where the authors considered the resulting torque, $\tau$, to be responsible for the rotational movement of the measured pendulum system:

$$
\tau=\tau_{T}-\tau_{F}=I \alpha_{0} .
$$

Here, $\tau_{F}$ is the torque of the friction force acting on the rotating shaft, while $I$ and $\alpha_{0}$ are the $M I$ and angular acceleration of the pendulum, respectively. It must be remembered that the torque of the traction force is $\tau_{T}=m\left(g-\alpha_{0} r_{p}\right) r_{p}$, where $r_{p}=(18.75 \pm 0.03) 10^{-3} \mathrm{~m}$ and $g=(9.79 \pm 0.01) \mathrm{m} / \mathrm{s}^{2}$. By means of Eq. (14), it can be shown that the inclination $\left(\Delta \tau_{T} / \Delta \alpha_{0}\right)$ is equal to $I$.

\section{Experimental results}

\subsection{Measure of torsion pendulum's rotational inertia}

The results of the torque applied to the torsion pendulum, $\tau_{T}$, by means of a traction wire, with one end tied to a pulley of radius $18.75 \mathrm{~mm}$ (Fig. 1(3)) and the angular acceleration, $\alpha_{0}$, using a rotary motion sensor; see Refs. $[15,16]$, are used to construct the Fig. 3. The inclinations obtained by a linear fit, provide the values $I$. 
Table 1. Experimental result, cp Ti: $\mathrm{d}=3.20 \pm 0.05 \mathrm{~mm}, \mathrm{~L}=17.50 \pm 0.05 \mathrm{~mm}$.

\begin{tabular}{c|c|c|c|c}
\hline $\begin{array}{c}I\left(\mathrm{kgm}^{2}\right. \\
\left.\mathrm{x} 10^{-4}\right)\end{array}$ & $100.6 \pm 0.7$ & $214 \pm 1$ & $399.6 \pm 0.8$ & $537 \pm 1$ \\
\hline$G(\mathrm{Gpa})$ & $30 \pm 4$ & $31 \pm 4$ & $30 \pm 4$ & $31 \pm 4$ \\
\hline$f_{0}(\mathrm{~Hz})$ & 6.70 & 4.62 & 3.35 & 2.91 \\
\hline$\delta$ & $\begin{array}{c}0.033 \\
\pm 0.001\end{array}$ & $\begin{array}{c}0.0304 \\
\pm 0.0004\end{array}$ & $\begin{array}{c}0.0322 \\
\pm 0.0006\end{array}$ & $\begin{array}{c}0.039 \\
\pm 0.002\end{array}$ \\
\hline
\end{tabular}

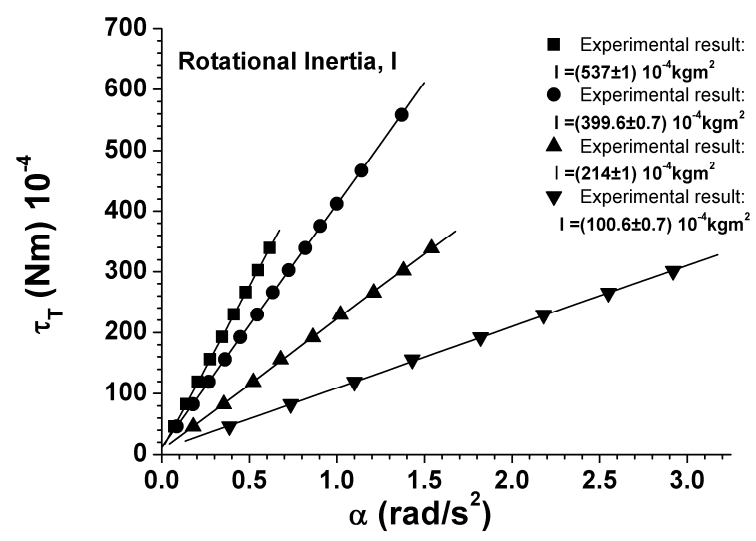

Fig. 3. Values of $I$ determined through linear fit with the experimental results. Four configurations were used with different positions and masses of the pendulum, corresponding to the four values of $I$.

\subsection{Validating the equation to determine the torsion modulus}

We deduced Eq. (9) in Sec. 2.1, which will be the object of study and whose validity is to be verified in the following sections. First, we chose a material interest, commercially pure titanium, cp Ti, and then we fixed a diameter of $3.20 \mathrm{~mm}$ For each fixed $L$ (six $L$ values were used), we changed the values of $I$ (the four $I$ values of item 3.1) by altering the pendulum configuration. Then we determined $G$, the oscillation frequency, $f_{0}$, and the parameter, $\delta$, to calculate the total friction of the pendulum, which is $Q_{T}^{-1}=\delta / 2 \pi$ (see Tables 1-6). This last parameter represents the sum of the internal friction of the sample cp $\mathrm{Ti}$ and friction bearings and means the air; for this reason, we used the total friction denomination. Further details and considerations have been extracted from Ref. [17]. With this system, it is possible to evaluate the interference of the sample and means (air and bearing) to which the torsion pendulum oscillates, but these effects cannot be determined separately [17]. To make it possible to eliminate the bearing and air, the same traditional method can be employed as that used to study the structural properties of the samples with mechanical spectroscopy. In this situation, a vacuum system must be added to the measuring equipment and the bearing removal. In this case, the system becomes more unstable mechanically and more expensive.

\subsubsection{Varying the length with a fixed diameter}

Tables 1-6 show the results for $\mathrm{cp} \mathrm{Ti}$, which were deduced by varying the length with fixed diameter.
Table 2. Experimental result, $c p$ Ti: $\mathrm{d}=3.20 \pm 0.05 \mathrm{~mm}, \mathrm{~L}=24.80 \pm 0.05 \mathrm{~mm}$.

\begin{tabular}{c|c|c|c|c}
\hline $\begin{array}{c}I\left(\mathrm{kgm}^{2}\right. \\
\left.\mathrm{x} 10^{-4}\right)\end{array}$ & $100.6 \pm 0.7$ & $214 \pm 1$ & $399.6 \pm 0.8$ & $537 \pm 1$ \\
\hline$G(\mathrm{Gpa})$ & $38 \pm 3$ & $38 \pm 3$ & $37 \pm 3$ & $38 \pm 3$ \\
\hline$f_{0}(\mathrm{~Hz})$ & 6.30 & 4.33 & 3.13 & 2.73 \\
\hline$\delta$ & 0.0314 & 0.033 & 0.036 & 0.0338 \\
\pm 0.0009 & \pm 0.001 & \pm 0.001 & \pm 0.0006 \\
\hline
\end{tabular}

Table 3. Experimental result, cp Ti: $d=3.20 \pm 0.05 \mathrm{~mm}, \mathrm{~L}=37.65 \pm 0.05 \mathrm{~mm}$.

\begin{tabular}{c|c|c|c|c}
\hline $\begin{array}{c}I\left(\mathrm{kgm}^{2}\right. \\
\left.\mathrm{x} 10^{-4}\right)\end{array}$ & $100.6 \pm 0.7$ & $214 \pm 1$ & $399.6 \pm 0.8$ & $537 \pm 1$ \\
\hline$G(\mathrm{Gpa})$ & $38 \pm 2$ & $37 \pm 2$ & $37 \pm 2$ & $37 \pm 2$ \\
\hline$f_{0}(\mathrm{~Hz})$ & 5.08 & 3.48 & 2.52 & 2.19 \\
\hline$\delta$ & 0.062 & $\begin{array}{c}0.060 \\
\pm 0.002\end{array}$ & $\begin{array}{c}0.0540 \\
\pm 0.0004\end{array}$ & $\begin{array}{c}0.056 \\
\pm 0.001\end{array}$ \\
\hline
\end{tabular}

Table 4. Experimental result, cp Ti: $\mathrm{d}=3.20 \pm 0.05 \mathrm{~mm}, \mathrm{~L}=45.30 \pm 0.05 \mathrm{~mm}$.

\begin{tabular}{c|c|c|c|c}
\hline $\begin{array}{c}I\left(\mathrm{kgm}^{2}\right. \\
\left.\mathrm{x} 10^{-4}\right)\end{array}$ & $100.6 \pm 0.7$ & $214 \pm 1$ & $399.6 \pm 0.8$ & $537 \pm 1$ \\
\hline$G(\mathrm{Gpa})$ & $37 \pm 2$ & $37 \pm 2$ & $36 \pm 2$ & $36 \pm 2$ \\
\hline$f_{0}(\mathrm{~Hz})$ & 4.61 & 3.15 & 2.28 & 1.98 \\
\hline$\delta$ & 0.0515 & $\begin{array}{c}0.052 \\
\pm 0.0004\end{array}$ & $\begin{array}{c}0.0517 \\
\pm 0.0003\end{array}$ & $\begin{array}{c}0.055 \\
\pm 0.001\end{array}$ \\
\hline
\end{tabular}

Table 5. Experimental result, cp Ti: $d=3.20 \pm 0.05 \mathrm{~mm}, \mathrm{~L}=61.00 \pm 0.05 \mathrm{~mm}$.

\begin{tabular}{c|c|c|c|c}
\hline $\begin{array}{c}I\left(\mathrm{kgm}^{2}\right. \\
\left.\mathrm{x} 10^{-4}\right)\end{array}$ & $100.6 \pm 0.7$ & $214 \pm 1$ & $399.6 \pm 0.8$ & $537 \pm 1$ \\
\hline$G(\mathrm{Gpa})$ & $43 \pm 2$ & $43 \pm 2$ & $42 \pm 1$ & $42 \pm 2$ \\
\hline$f_{0}(\mathrm{~Hz})$ & 4.26 & 2.91 & 2.12 & 1.83 \\
\hline$\delta$ & 0.0459 & $\begin{array}{c}0.0419 \\
\pm 0.0004\end{array}$ & $\begin{array}{c}0.0396 \\
\pm 0.0004\end{array}$ & $\begin{array}{c}0.0338 \\
\pm 0.0005\end{array}$ \\
\hline
\end{tabular}

Table 6. Experimental result, cp Ti: $\mathrm{d}=3.20 \pm 0.05 \mathrm{~mm}, \mathrm{~L}=72.40 \pm 0.05 \mathrm{~mm}$.

\begin{tabular}{c|c|c|c|c}
\hline $\begin{array}{c}I\left(\mathrm{kgm}^{2}\right. \\
\left.\mathrm{x} 10^{-4}\right)\end{array}$ & $100.6 \pm 0.7$ & $214 \pm 1$ & $399.6 \pm 0.8$ & $537 \pm 1$ \\
\hline$G(\mathrm{Gpa})$ & $47 \pm 1$ & $47 \pm 1$ & $46 \pm 1$ & $48 \pm 1$ \\
\hline$f_{0}(\mathrm{~Hz})$ & 4.12 & 2.82 & 2.04 & 1.79 \\
\hline$\delta$ & $\begin{array}{c}0.0185 \\
\pm 0.0001\end{array}$ & $\begin{array}{c}0.01802 \\
\pm 0.00007\end{array}$ & $\begin{array}{c}0.0181 \\
\pm 0.0002\end{array}$ & $\begin{array}{c}0.0194 \\
\pm 0.0004\end{array}$ \\
\hline
\end{tabular}

These results in these tables are presented in Fig. 4.

The experimental points and curves that resulted from the linear fit (see Fig. 4) provided a way to verify the validity of Eq. (9). In this case, a fixed diameter was used for the sample because the influence of the parameter $\delta$ was very small compared to $I$ in the calculus of $G$. This latter assertion is discussed in Sec. 3.2.4. By calculating the proportion between the moments of inertia used, $I$, and their inclinations, $B$, we can check whether they are the same. If this is true, we show that Eq. (9) describes $G$ for the considered interval of $L$, that is, 17.50 to $72.40 \mathrm{~mm}$. 
Table 7. Proportions of experimental values of $I$ and $B$ with the respective percentage deviation between them, with fixed $d$ and varying $L$.

\begin{tabular}{c|c|c}
\hline Proportion between $I$ & Proportion between $B$ & $\begin{array}{c}\text { Percentage deviation } \\
(\%)\end{array}$ \\
\hline $214 / 100.6=2.13$ & $81 / 39=2.08$ & -2.35 \\
\hline $399.6 / 214=1.87$ & $152 / 81=1.88$ & 0.535 \\
\hline $537 / 399.6=1.34$ & $205 / 152=1.35$ & 0.746 \\
\hline $399.6 / 100.6=3.97$ & $152 / 39=3.90$ & -1.76 \\
\hline $537 / 214=2.51$ & $205 / 81=2.53$ & 0.797 \\
\hline $537 / 100.6=5.34$ & $205 / 39=5.26$ & -1.50 \\
\hline
\end{tabular}

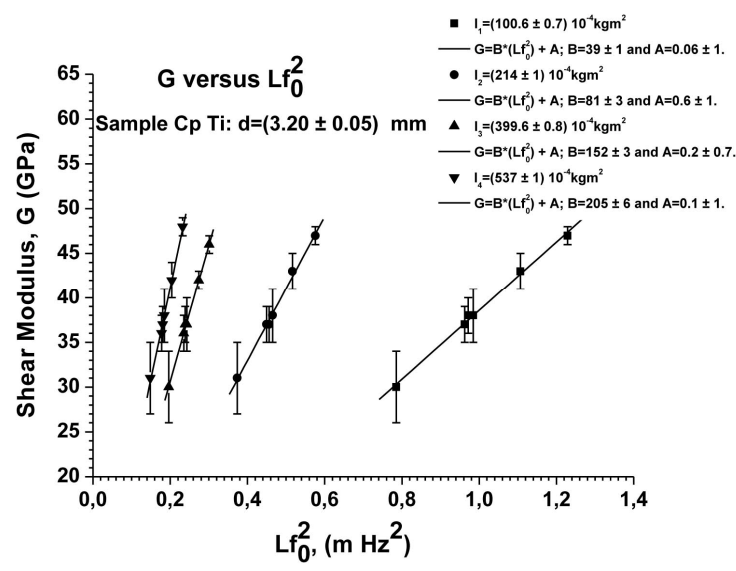

Fig. 4. Curves of $G$ in function $L f_{0}^{2}$. For each fixed rotational inertia, $I$, the length, $L$, and frequency, $f_{0}$, are altered. The angular coefficient of the linear fit, such as the diameter of the sample, was set, depending only on the parameters $\delta$ and $I$.

The table below provides these proportions and the percentages of deviation between them.

The $I$ values in Sec. 3.1 were determined independently of $G$ in Eq. (9). However, as can be seen in Table 7, the ratio between the values of $G$, valid for the established experimental conditions, should reflect the ratio between the values of $I$, if the parameter $\delta$ does not interfere significantly. This should occur since the percentage deviations were small. In this case, Eq. (9) can be used to determine $G$.

\subsubsection{Frequency in the function of the rotational inertia for varying lengths and fixed diameters}

The oscillation frequency, $f_{0}$, changes with the values of inertia, $I$, as shown in Fig. 5. For fixed values of $I$, the $f_{0}$ value decreases by increasing the length, $L$, of the sample and keeping $d$ unchanged. This could not have happened if a sample had been used in which the internal structure was modified.

\subsubsection{Values of the torsion modulus for different lengths and fixed diameters}

Fig. 6 shows that $G$ depends on the length of the sample, $L$, as was expected based on Eq. (9). Another important fact is that $G$ is independent of the $I$ values because these $G$ values are practically the same as considering the error associated with each of them. In the Ref. [21], the modulus of elasticity,

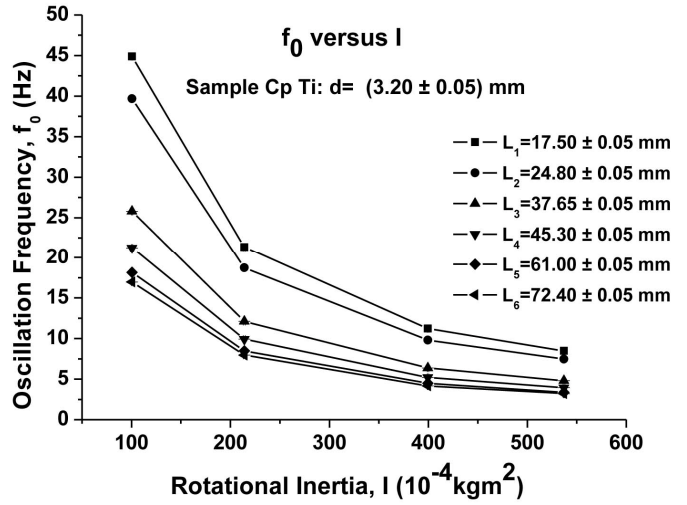

Fig. 5. Oscillation frequency as a function of rotational inertia, $I$, with a fixed and constant diameter sample.

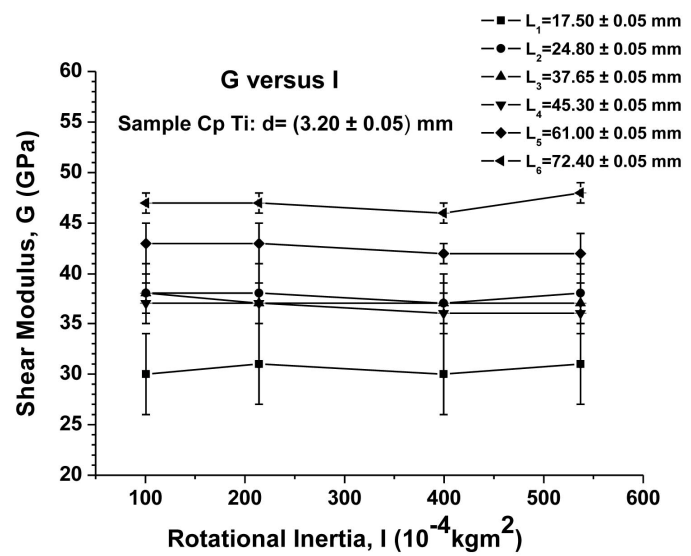

Fig. 6. Experimental values of $G$ in function of $I$ to $\mathrm{cp}$ Ti samples, with diameter $3.20 \mathrm{~mm}$ and $L$ varying in the interval of $17.50 \mathrm{~mm}$ to $72.40 \mathrm{~mm}$.

$E$, for a commercially pure titanium sample, has shown results in an interval of $100 \mathrm{GPa}$ to $110 \mathrm{GPa}$. This value can be obtained by means of $G$ if we use the equation that takes into account the Poisson coefficient, $v$, of the elasticity theory; this is $v=E / 2 G-1$. For an $L$ between $24.50 \mathrm{~mm}$ and 45.30 $\mathrm{mm}$, wherein $G$ has approximately the same value, $G=37 \pm 2$ $\mathrm{GPa}$, using the Poisson ratio, $v=0.33$, we find a value for $E$ of $98 \pm 2 \mathrm{GPa}$. Even though this value is below the lower limit, if we consider its error, it is still within the interval of $100 \mathrm{GPa}$ to $110 \mathrm{GPa}$. For $L=61.00 \mathrm{~mm}$, using the same reasoning as before, we find $E=113 \pm 2 \mathrm{GPa}$, in which case it is slightly higher than the value of the upper limit, $E$. For $L=17.50 \mathrm{~mm}$ and $L=72.40 \mathrm{~mm}$, respectively, we found that $E$ is equal to $81 \pm 4 \mathrm{GPa}$ and $125 \pm 1 \mathrm{GPa}$ for each of the sample lengths. In this case, the values are outside the reference range [21]. It should be recalled that $v=0.33$ is of questionable value because it is known that this value varies from 0.31 to 0.36 [22], and it depends upon the degree of purity of the cp Ti sample used. Average values of $E$ for cp Ti, which were obtained by either nanoindentation or ultrasonic techniques, showed values of $119 \pm 4.6 \mathrm{GPa}$ and $121 \pm 0.87 \mathrm{GPa}$, respectively [23]. This indicates that the values of $E$ may be different, depending on the measurement technique used. In reference 


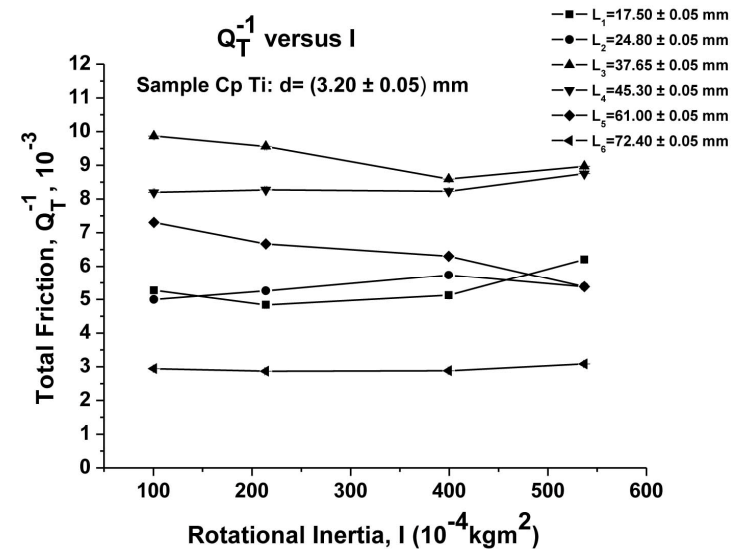

Fig. 7. Values of $Q_{T}^{-1}$ in the function of rotational inertia, $I$, to cp Ti samples of diameter $3.20 \mathrm{~mm}$, and $L$ varying from $17.50 \mathrm{~mm}$ to $72.40 \mathrm{~mm}$.

[22], which provides values for titanium alloys, $G$ values are tabulated between $39 \mathrm{GPa}$ and $44 \mathrm{GPa}$, and $E$ between 100 $\mathrm{GPa}$ and $120 \mathrm{GPa}$. Given that we used a different technique from those mentioned above, it is perfectly understandable that the values found here would be slightly different from those in the Ref. [21]. In our case, the samples with the shortest length $(17.50 \mathrm{~mm})$ and those with the longest length $(72.40 \mathrm{~mm})$ were the ones with the greatest differences in relation to the literature.

\subsubsection{Total friction with fixed diameters and varying lengths}

Fig. 7 represents the total internal friction as a function of the rotational inertia of the torsion pendulum, $I$. Sec. 3.2.1 affirms that $\delta$ should not influence the values of $G$ in a significant way, and this may be seen from the results in Fig. 6. We use the expression $Q_{T}^{-1}=\delta / 2 \pi$ to calculate the total friction of the pendulum. When calculating $G$ using Eq. (9), we have the factor, $\left(1-\delta^{2} / 4 \pi^{2}\right)$, which depends on $Q_{T}^{-1}$. Considering $Q_{T}^{-1}$ to be the highest value - that is, $10 \times 10^{-3}$ (see Fig. 7) - and calculating that factor, we find 0.9999 . Note that in terms of percentage, this modifies the value of $G$ to become $0.01 \%$. So in fact, the influence of $\delta$ is not significant in the value of $G$ determined in this work.

\subsubsection{Varying the diameter and maintaining the fixed length for each rotational inertia}

The tables below present the experimental results of the $\mathrm{cp}$ Ti samples for four different diameters while maintaining the length, $L$, at $33.45 \mathrm{~mm}$. Since our goal is to check Eq. (9), we use four values, $I$, (the same as those used in Sec. 3.1) to change the value of $f_{0}$.

Figs. 8-11 were constructed based on Tables 8-11, such that a linear fit was carried out on the experimental data for each $I$ studied.

The experimental data and the experimental curves with the appropriate linear fit (see Figs. 8-11) was a way to verify the validity of Eq. (9). In this case, different diameters with fixed $L$ values were used because the influence of the parameter $\delta$
Table 8. Experimental result, cp Ti: $d=3.20 \pm 0.05 \mathrm{~mm}, L=33.45 \pm 0.05 \mathrm{~mm}$

\begin{tabular}{c|c|c|c|c}
\hline $\begin{array}{c}I\left(\mathrm{kgm}^{2}\right. \\
\left.\mathrm{x} 10^{-4}\right)\end{array}$ & $100.6 \pm 0.7$ & $214 \pm 1$ & $399.6 \pm 0.8$ & $537 \pm 1$ \\
\hline$G(\mathrm{Gpa})$ & $41 \pm 3$ & $41 \pm 3$ & $41 \pm 3$ & $41 \pm 3$ \\
\hline$f_{0}(\mathrm{~Hz})$ & 5.63 & 3.86 & 2.82 & 2.44 \\
\hline$\delta$ & 0.0181 & 0.0168 & 0.022 & 0.030 \\
\pm 0.0003 & \pm 0.0004 & \pm 0.0006 & \pm 0.001 \\
\hline
\end{tabular}

Table 9. Experimental result, cp Ti: $d=3.80 \pm 0.05 \mathrm{~mm}, L=33.45 \pm 0.05 \mathrm{~mm}$.

\begin{tabular}{c|c|c|c|c}
\hline $\begin{array}{c}I\left(\mathrm{kgm}^{2}\right. \\
\left.\mathrm{x} 10^{-4}\right)\end{array}$ & $100.6 \pm 0.7$ & $214 \pm 1$ & $399.6 \pm 0.8$ & $537 \pm 1$ \\
\hline$G(\mathrm{Gpa})$ & $38 \pm 2$ & $38 \pm 2$ & $37 \pm 2$ & $38 \pm 2$ \\
\hline$f_{0}(\mathrm{~Hz})$ & 7.64 & 5.24 & 3.79 & 3.29 \\
\hline$\delta$ & 0.021 & 0.0270 & 0.030 & 0.030 \\
\pm 0.001 & \pm 0.0003 & \pm 0.001 & \pm 0.001 \\
\hline
\end{tabular}

Table 10. Experimental result, cp Ti: $d=4.70 \pm 0.05 \mathrm{~mm}, L=33.45 \pm$ $0.05 \mathrm{~mm}$.

\begin{tabular}{c|c|c|c|c}
\hline $\begin{array}{c}I\left(\mathrm{kgm}^{2}\right. \\
\left.\mathrm{x} 10^{-4}\right)\end{array}$ & $100.6 \pm 0.7$ & $214 \pm 1$ & $399.6 \pm 0.8$ & $537 \pm 1$ \\
\hline$G(\mathrm{Gpa})$ & $32 \pm 1$ & $32 \pm 1$ & $32 \pm 1$ & $32 \pm 1$ \\
\hline$f_{0}(\mathrm{~Hz})$ & 10.77 & 7.36 & 5.36 & 4.65 \\
\hline$\delta$ & 0.023 & 0.0189 & 0.025 & 0.0191 \\
& \pm 0.004 & \pm 0.0004 & \pm 0.001 & \pm 0.0003 \\
\hline
\end{tabular}

Table 11. Experimental result, cp Ti: $d=5.00 \pm 0.05 \mathrm{~mm}, L=33.45 \pm$ $0.05 \mathrm{~mm}$.

\begin{tabular}{c|c|c|c|c}
\hline $\begin{array}{c}I\left(\mathrm{kgm}^{2}\right. \\
\left.\times 10^{-4}\right)\end{array}$ & $100.6 \pm 0.7$ & $214 \pm 1$ & $399.6 \pm 0.8$ & $537 \pm 1$ \\
\hline$G(\mathrm{Gpa})$ & $31 \pm 1$ & $31 \pm 1$ & $31 \pm 1$ & $31 \pm 1$ \\
\hline$f_{0}(\mathrm{~Hz})$ & 12.00 & 8.23 & 5.98 & 5.19 \\
\hline$\delta$ & $\begin{array}{c}0.0210 \\
\pm 0.0002\end{array}$ & $\begin{array}{c}0.0207 \\
\pm 0.0003\end{array}$ & $\begin{array}{c}0.0218 \\
\pm 0.0009\end{array}$ & $\begin{array}{c}0.0180 \\
\pm 0.0002\end{array}$ \\
\hline
\end{tabular}

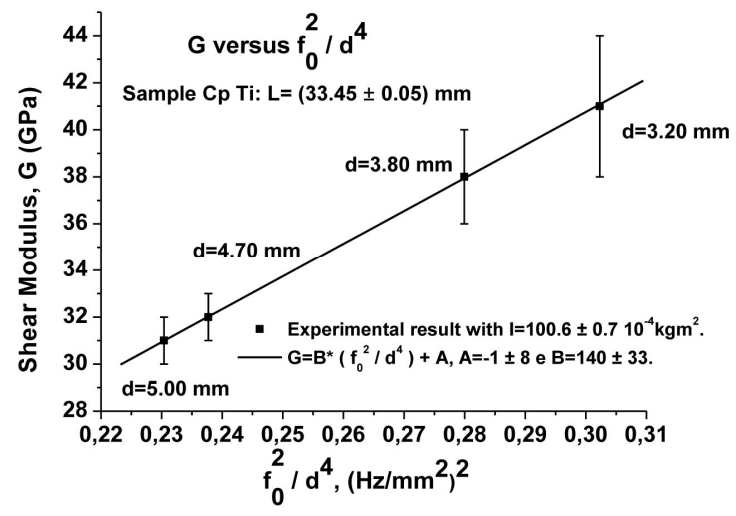

Fig. 8. Values of $G$ in function of the relation $\left(f_{0}\right)^{2} / d^{4}$, to cp Ti samples, with $L=33.45 \mathrm{~mm}$ and diameters $3.20 \mathrm{~mm}, 3.80 \mathrm{~mm}, 4.70 \mathrm{~mm}$ and $5.00 \mathrm{~mm}$. Rotational inertia of $I=(100.6 \pm 0.7) 10^{-4} \mathrm{kgm}^{2}$ was used.

was very small, compared to $I$, for calculating $G$. This last statement is discussed in Sec. 3.2.6.

Calculating the proportion between the moment of inertia 


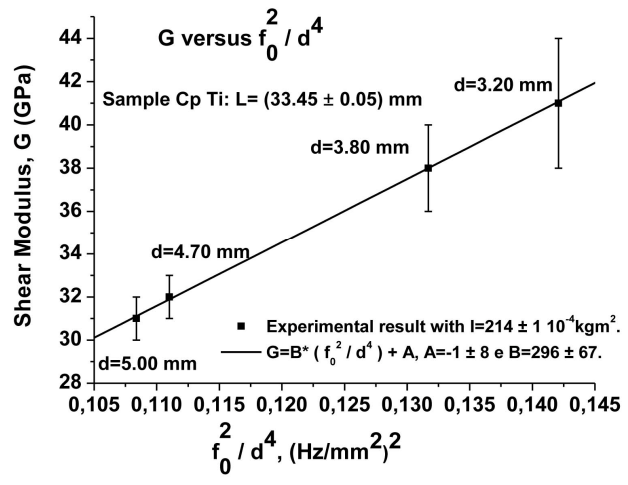

Fig. 9. Values of $G$ in function of the relation $\left(f_{0}\right)^{2} / d^{4}$, to cp Ti samples, with $L=33.45 \mathrm{~mm}$ and diameters $3.20 \mathrm{~mm}, 3.80 \mathrm{~mm}, 4.70 \mathrm{~mm}$ and $5.00 \mathrm{~mm}$. Rotational inertia of $I=(214 \pm 1) 10^{-4} \mathrm{kgm}^{2}$ was used.

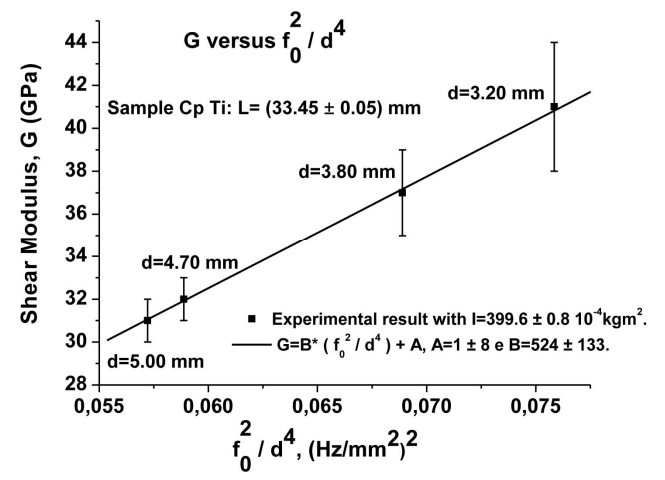

Fig. 10. Values of $G$ in function of the relation $\left(f_{0}\right)^{2} / d^{4}$, to cp Ti samples, with $L=33.45 \mathrm{~mm}$ and diameters $3.20 \mathrm{~mm}, 3.80 \mathrm{~mm}, 4.70 \mathrm{~mm}$ and $5.00 \mathrm{~mm}$. Rotational inertia of $I=(399.6 \pm 0.8) 10^{-4} \mathrm{kgm}^{2}$ was used.

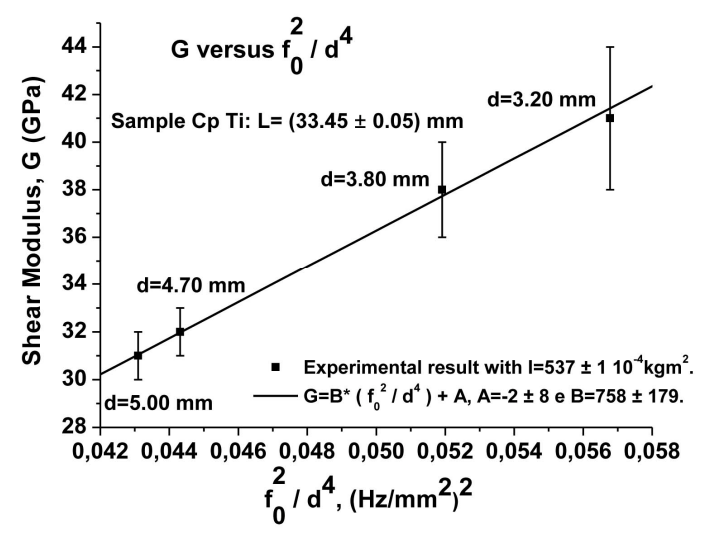

Fig. 11. Values of $G$ in function of the relation $\left(f_{0}\right)^{2} / d^{4}$, to cp Ti samples, with $L=33.45 \mathrm{~mm}$ and diameters $3.20 \mathrm{~mm}, 3.80 \mathrm{~mm}, 4.70 \mathrm{~mm}$ and $5.00 \mathrm{~mm}$. Rotational inertia of $I=(537 \pm 1) 10^{-4} \mathrm{kgm}^{2}$ was used.

used, $I$, and the related inclinations, $B$, we can check to see whether they are the same. If this is the case, we show that Eq. (9) describes $G$ for the studied interval of $L$, that is, $17.50 \mathrm{~mm}$ to $72.40 \mathrm{~mm}$. It is observed that the error associated with the linear coefficient is always greater than the setting value, which means that it can be discarded and the adjusted line passes through the origin. This confirms that Eq. (9) really
Table 12. Proportions of experimental values of $I$ and $B$ with the respective percentage deviation between them, with fixed $L$ and by varying $d$.

\begin{tabular}{c|c|c}
\hline Proportion between $I$ & Proportion between $B$ & $\begin{array}{c}\text { Percentage } \\
\text { deviation (\%) }\end{array}$ \\
\hline $214 / 100.6=2.13$ & $296 / 140=2.11$ & -0.939 \\
\hline $399.6 / 214=1.87$ & $524 / 296=1.77$ & -5.35 \\
\hline $537 / 399.6=1.34$ & $758 / 524=1.45$ & 8.21 \\
\hline $399.6 / 100.6=3.97$ & $524 / 140=3.74$ & -5.79 \\
\hline $537 / 214=2.51$ & $758 / 296=2.56$ & 1.99 \\
\hline $537 / 100.6=5.34$ & $758 / 140=5.41$ & 1.31 \\
\hline
\end{tabular}

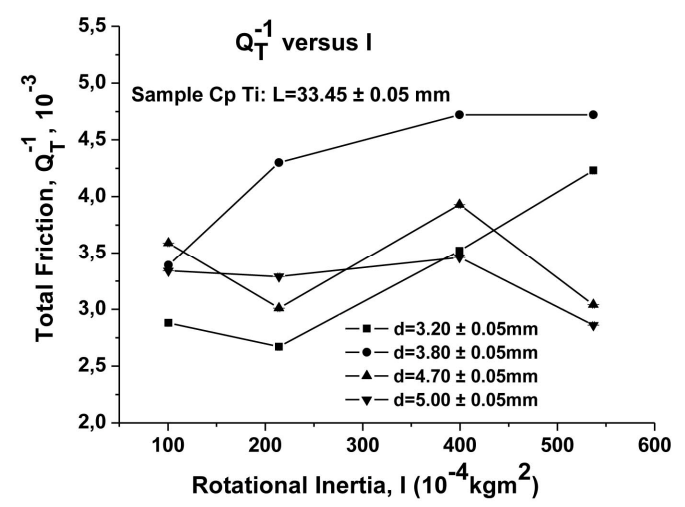

Fig. 12. Values of $Q_{T}^{-1}$ in the function of rotational inertia, $I$, to cp Ti samples with $\mathrm{L}=33.45 \mathrm{~mm}$ and diameters of $3.20 \mathrm{~mm}, 3.80 \mathrm{~mm}, 4.70$ $\mathrm{mm}$ and $5.00 \mathrm{~mm}$.

passes through the origin.

The table below provides these proportions and the percentages of deviation between them.

The values of $I$ in Sec. 3.1 determined $G$ in an independent manner by means of Eq. (9); however, as can be seen in Table 12 , the proportions between the values of $G$ should be valid for established experimental conditions and should reflect the ratio between values of $I$ if the parameter $\delta$ does not interfere significantly. Sec. 3.2.6 shows that the percentage deviations in Table 12 are not due to $\delta$ parameters. As can be seen from the linear fits in Figs. 8-11, the values of the inclination, $B$, and its associated errors are greater than the errors found in Sec. 3.2.1. This explains and justifies the greatest deviations found. In this case, it is confirmed that Eq. (9) can be used to determine $G$.

\subsubsection{Total friction with fixed lengths and varying diameters}

Fig. 12 represents the total internal friction, $Q_{T}^{-1}$, as a function of the rotational inertia of the torsion pendulum, $I$. In Secs. 3.2.1 and 3.2.4, we stated that $\delta$ does not influence the value of $G$ in a significant way, and this can be observed in the results shown in Fig. 11. For the calculation of the total friction of the pendulum, we use the expression, $Q_{T}^{-1}=\delta / 2 \pi$. In the calculations of $G$, using Eq. (9), we have the factor $\left(1-\delta^{2} / 4 \pi^{2}\right)$, which depends on $Q_{T}^{-1}$. By considering the highest value of $Q_{T}^{-1}$, which is $5.0 \times 10^{-3}$, and calculating that 


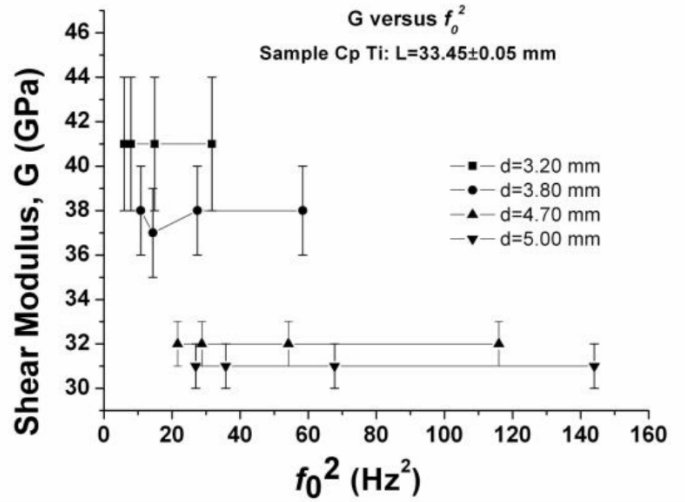

Fig. 13. Values of $G$ in function of $\left(f_{0}\right)^{2}$ to cp Ti samples with $L=$ $33.45 \mathrm{~mm}$ and diameters of $3.20 \mathrm{~mm}, 3.80 \mathrm{~mm}, 4.70$ and $5.00 \mathrm{~mm}$.

factor, we find 0.99998 . Note that this modifies the value of $G$ to become $0.001 \%$ in percentage terms. Therefore, the interference of $Q_{T}^{-1}$ is not significant in the value of $G$.

\subsubsection{Torsion modulus as a function of $\left(f_{0}\right)^{2}$ to fixed lengths and varying diameters}

Fig. 13 shows that when the length of the sample remains unchanged, $G$ decreases as the values of the diameters increase. For the $3.20 \mathrm{~mm}$ and $3.80 \mathrm{~mm}$ diameters, respectively, using the same reasoning as in Sec. 3.2.3, with $v=0.33$, we come to $E$ being equal to $109 \mathrm{GPa}$ and $101 \mathrm{GPa}$. For the diameters $4.70 \mathrm{~mm}$ and $5.00 \mathrm{~mm}$, the values are below $100 \mathrm{GPa}$; therefore, they are $85 \mathrm{GPa}$ and $83 \mathrm{GPa}$, respectively. This shows that $E$ decreases as $d$ increases. We observe that the $G$ values do not depend on the frequency squared. Since the $f_{0}$ frequency is related to the rotational inertia of the torsion pendulum (see Fig. 5), they experience changes in oscillation frequency so that $G$ becomes unaltered, as was expected. This was probably due to the increasing value of $I$ and samples that maintain the same internal structure. This can be seen in Fig. 13.

\section{Conclusions}

The presentation of this measurement technique and method, plus the fact that it obtained excellent results in this work, reinforces it as an alternative way to study the elastic modulus of the torsion of titanium and its alloys. The experimental validation of the equation deduced through mechanical spectroscopy studies and the theory of relaxation was a way to verify the proposed method. According to the results, this alternative experiment is different from others that have been used to determine the $G$ modulus. Therefore, this research can be used in the study of mechanical properties of materials.

\section{Acknowledgment}

The authors thank the Brazilian agency, FAPESP, process number: 2007/04094-9, and Capes, process number: BEX 6571/14-0.

\section{Nomenclature}

G : Torsion or shear modulus

$Q_{T}^{-1} \quad$ : Total internal friction

I : Rotational inertia

$G_{0} \quad$ : Torsion constant

$\theta \quad$ : Angular position

$\theta_{0} \quad$ : Initial amplitude

$\omega_{0} \quad$ : Angular frequency

$f_{0} \quad$ : Oscillation frequency

$r_{p} \quad:$ Radius of the pulley

$R \quad$ : Radius of the sample

$d \quad$ : Diameter of the sample

$F \quad:$ Force

cp Ti : Commercially pure titanium

$T \quad$ : Period

$t_{0} \quad:$ Parameter from the linear fit corresponding to $T$

$W \quad$ : Parameter corresponding to $\omega_{0}$

$t_{C} \quad$ : Parameter from the linear fit program

$A \quad$ : Parameter from the linear fit, amplitude

$\tau_{T} \quad:$ Torque of the traction force

$\tau_{F} \quad:$ Torque of the friction force

$\alpha_{0} \quad$ : Angular acceleration

$m$ : Mass

$B \quad$ : Angular coefficient or inclination

\section{References}

[1] D. Banerjee and J. C. Williams, Perspectives on titanium science and technology, Acta Materialia, 61 (2013) 844-879.

[2] M. Niinomi, M. Nakai and J. Hieda, Development of new metallic alloys for biomedical applications, Acta Biomaterialia, 8 (11) (2012) 3888-3903.

[3] T. Hanawa, Research and development of metals for devices based on clinical needs, Science and Technology of Advanced Materials, 11 (6) (2012) 64102-64116.

[4] M. Long and H. J. Rack, Titanium alloys in total joint replacement-a materials science perspective, Biomaterials, 19 (1998) 1621-1639.

[5] M. Geetha, A. K. Singh, R. Asokamani and A. K. Gogia, Ti based biomaterials, the ultimate choice for orthopaedic implants - A review, Progress in Materials Science, 54 (2009) 397-425.

[6] R. Truel, C. Elbaum and B. B. Chick, Ultrasonic methods in solid state physics, Academic Press: New York (1969) 136138.

[7] B. D. Ratner, A. S. Hoffman, F. J. Schoen and J. E. Lemons, An introduction to materials in medicine, Biomaterials Science, Academic Press: New York (2004).

[8] A. S. Nowick and B. S. Berry, Anelastic relaxation in crystalline solids, Academic Press, New York (1972).

[9] F. A. Tyler, A Laboratory Manual of Physics, 4th Ed., Edward Arnold: London (1974) 22-24.

[10] J. Goldemberg, Fisica Geral e Experimental, 2nd Ed., Companhia Editora Nacional: São Paulo (1970) 481-483. 
[11] P. F. Hinrichsen, Bifilar suspension measurement of boat inertia parameters, Journal of Sailboat Technology, 1 (2014) $1-37$.

[12] D. Amrani, Computerized rotational system to study the moment of inertia of different objects, Eur. J. Phys., 27 (2006) 1063-1069.

[13] T. Eadkhong, R. Rajsadorn, P. Jannual and S. Danworaphong, Rotational dynamics with tracker, Eur. J. Phys., 33 (2012) 615-622.

[14] I. W. Griffiths, J. Watkins and D. Sharpe, Measuring the moment of inertia of the human body by a rotating platform method, Am. J. Phys., 73 (2005) 85-92.

[15] C. A. F. Pintão, M. P. de Souza Filho, C. R. Grandini and R. Hessel, Medida do momento de inércia disco, Rev. Bras. Ens. Fis., 23 (2001) 48-52.

[16] C. A. F. Pintão, M. P. de Souza Filho, C. R. Grandini and R. Hessel, Experimental study of the conventional equation to determine plate's moment of inertia, Eur. J. Phys., 25 (2004) 409-417.

[17] C. A. F. Pintão, Measurement of the rotational inertia of bodies by using mechanical spectroscopy, J. Mech. Sci. Tech. (Seoul. Print), 28 (10) (2014) 4011-4020.

[18] S. P. Timoshenko and J. N. Goodier, Teoria da elasticidade,

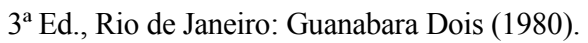

[19] I. H. Shames and F. A. Cozzareli, Elastic and inelastic Stress analysis, Prentice-Hall International, Englewood Cliffs, New Jersey (1992).
[20] A. P. Boresi and K. P. Chong, Elasticity in engineering mechanics, $2^{\mathrm{a}}$ Ed., John Wiley \& Sons (1999).

[21] H. E. Boyer and T. L. Gal, Metal Handbook, ASM, Ohio (1985).

[22] S. P. Timoshenko and J. M. Gere, Mechanics of Materials, Van Nostrand Reinhold Co., New York (1972).

[23] P. Majundar, S. B. Singh and M. Chakraborty, Elastic modulus of biomedical titanium alloys by nano-indentation and ultrasonic techniques - A comparative study, Mater. Sci. Eng., A489 (2008) 419-425.

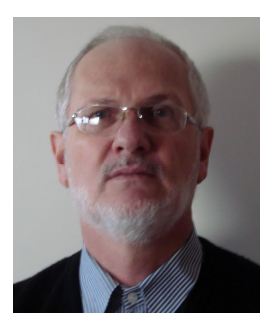

Carlos Alberto Fonzar Pintão was born in Bilac, Brazil in 1954. He received B.Sc. degree in Physics and Mechanical Engineering from the São Paulo University (USP) and the Faculty of Industrial Engineering (FEI), Brazil in 1985 and 1979, respectively, the M.Sc. degree from the São Paulo University (USP), São Carlos, Brazil in 1989 and the Ph.D. degree from the São Paulo University (USP), São Carlos, Brazil in 1996. During the last 29 years he has lectured Physics for Engineering and Physics courses. Most of his research is focused on electron secondary emission in metals and polymers, mechanical properties of materials (elastic modulus and internal friction) and physics experiments in education. 